\title{
Feeding Cues Alter Clock Gene Oscillations and Photic Responses in the Suprachiasmatic Nuclei of Mice Exposed to a Light/Dark Cycle
}

\author{
Jorge Mendoza, ${ }^{\star}$ Caroline Graff, ${ }^{\star}$ Hugues Dardente, Paul Pevet, and Etienne Challet \\ Laboratory of Neurobiology of Rhythms, Centre National de la Recherche Scientifique, Unité Mixte de Recherche 7518, Department of Neuroscience, \\ Institut Fédératif de Recherche 37, University Louis Pasteur, F-67084 Strasbourg, France
}

The suprachiasmatic nuclei (SCN) of the hypothalamus contain the master mammalian circadian clock, which is mainly reset by light. Temporal restricted feeding, a potent synchronizer of peripheral oscillators, has only weak influence on light-entrained rhythms via the $\mathrm{SCN}$, unless restricted feeding is coupled with calorie restriction, thereby altering phase angle of photic synchronization. Effects of daytime restricted feeding were investigated on the mouse circadian system. Normocaloric feeding at midday led to a predominantly diurnal (60\%) food intake and decreased blood glucose in the afternoon, but it did not affect the phase of locomotor activity rhythm or vasopressin expression in the SCN. In contrast, hypocaloric feeding at midday led to $2-4 \mathrm{~h}$ phase advances of three circadian outputs, locomotor activity rhythm, pineal melatonin, and vasopressin mRNA cycle in the SCN, and it decreased daily levels of blood glucose. Furthermore, Per1 and Cry2 oscillations in the SCN were phase advanced by 1 and $3 \mathrm{~h}$, respectively, in hypocalorie- but not in normocalorie-fed mice. The phase of Per2 and Bmall expression remained unchanged regardless of feeding condition. Moreover, the shape of behavioral phase-response curve to light and light-induced expression of Per1 in the SCN were markedly modified in hypocalorie-fed mice compared with animals fed ad libitum. The present study shows that diurnal hypocaloric feeding affects not only the temporal organization of the SCN clockwork and circadian outputs in mice under light/dark cycle but also photic responses of the circadian system, thus indicating that energy metabolism modulates circadian rhythmicity and gating of photic inputs in mammals.

Key words: behavior; circadian; feeding; gene; light; rhythm; suprachiasmatic

\section{Introduction}

In mammals, the suprachiasmatic nuclei (SCN) of the hypothalamus contain the master circadian clock that coordinates the daily temporal organization of physiology and behavior. The SCN clock, which generates circadian rhythms, is synchronized to cyclic environmental changes, mainly the light/dark cycle (LD) (Takahashi et al., 2001).

The molecular mechanisms underlying circadian rhythmicity involve self-sustaining transcriptional/translational feedback loops based on rhythmic expression of the mRNA and proteins of clock components. mRNA of three Period genes (Per1, Per2, and

\footnotetext{
Received June 9, 2004; revised Dec. 14, 2004; accepted Dec. 25, 2004

This work was funded in part by a "Crédit Exceptionnel pour Jeunes Équipes" from the Centre National de la Recherche Scientifique (E.C.). The plasmids for synthesis of $r$ Per1, $m$ Per2, and $m$ Cry 2 were generously donated by Dr. Hitoshi Okamura (Kobe University Graduate School of Medicine, Kobe, Japan). We are grateful to Christiane Calgari, Sylviane Gourmelen, and Dominique Streicher for excellent technical assistance. We thank Drs. David Hazlerigg and Maria-Teresa Romero for constructive comments.

*J.M. and C.G. contributed equally to this work.

Correspondence should be addressed to Etienne Challet, Centre National de la Recherche Scientifique, Unité Mixte de Recherche 7518, Université Louis Pasteur, 5 rue Blaise Pascal, F-67084 Strasbourg cedex, France. E-mail: challet@neurochem.u-strasbg.fr.

J. Mendoza's present address: Department of Anatomy, Faculty of Medicine, Universidad Nacional Autónoma de México, Mexico City DF 04510, Mexico.

H. Dardente's present address: Douglas Hospital Research Centre, McGill University, Montreal, Quebec H4H 1R3, Canada.

DOI:10.1523/JNEUROSCI.4397-04.2005

Copyright $\odot 2005$ Society for Neuroscience $\quad$ 0270-6474/05/251514-09\$15.00/0
}

Per3) oscillate with peak levels during daytime (Albrecht et al., 1997; Zylka et al., 1998). Transcription of Per, two Cryptochrome (Cry1 and Cry2) Rev-Erbo, and two Dec (Dec1 and Dec2) is activated by heterodimers of transcription factors, CLOCK and BMAL1 (Kume et al., 1999; Honma et al., 2002; Preitner et al., 2002). Whereas Clock mRNA levels do not markedly oscillate in mouse SCN neurons (Shearman et al., 2000), Bmall mRNA levels show daily variations with nocturnal peak and daytime trough (Abe et al., 1998). Several clock proteins, including CRY and DEC, inhibit transcription of Per and Cry through binding to, or competition with, CLOCK/BMAL1 (Kume et al., 1999; Honma et al., 2002). Moreover, Bmall transcription is repressed by REV$\mathrm{ERB} \alpha$ (Preitner et al., 2002) and activated by ROR $\alpha$ (Sato et al., 2004).

Synchronization of the SCN clock to light has been associated with increases of Per1 and Per2 mRNA (Albrecht et al., 1997, 2001; Shigeyoshi et al., 1997). Synchronizing effects of food availability differ greatly from photic phase resetting. Behavioral outputs of light-synchronized SCN are usually not affected by restricted feeding (Mistlberger, 1994; Stephan, 2002). Accordingly, daily food access limited to daytime does not change clock gene expression in the SCN of rodents exposed to LD (Damiola et al., 2000; Hara et al., 2001; Stokkan et al., 2001; Wakamatsu et al., 2001). When rats and C57BL mice kept under LD are fed daily with only a hypocaloric diet, however, significant phase advances 
of circadian rhythms of melatonin, body temperature, and locomotor activity have been detected (Challet et al., 1997, 1998), suggesting that the phase of the SCN is modulated by feedingrelated/metabolic cues.

Here we investigated the molecular mechanisms whereby timed calorie restriction alters the phase angle of photic synchronization. For this purpose, daily patterns of clock and clockcontrolled gene expression were determined in the SCN of mice fed during daytime with either hypocaloric or normocaloric diet and compared with ad libitum-fed control animals. Moreover, phase-shift responses of locomotor activity rhythm to light, lightinduced suppression of pineal melatonin, and light-induced expression of clock genes in the SCN were compared between hypocalorie-fed and ad libitum-fed mice.

\section{Materials and Methods}

Animal housing and experimental design. Male, 8-week-old C3H mice (Charles River, Lyon, France) were housed singly in cages equipped with a running wheel $(10 \mathrm{~cm}$ diameter $)$ in a room at $23 \pm 1^{\circ} \mathrm{C}$ with a $12 \mathrm{~h}$ light/dark cycle (lights on at 5:00 A.M.) for 2-3 weeks. Under LD conditions, times of day were converted to Zeitgeber times (ZT), in which ZT0 and ZT12 were the onsets of light and darkness, respectively. On the first day of constant darkness (DD), times of day were converted to projected ZT (pZT), in which pZT0 and pZT12 were defined as the respective projected time of lights on and lights off in the previous lighting cycle. During daytime, light intensity was $\sim 200$ lux at the level of the cages. Food pellets and water were available ad libitum unless otherwise stated. Food pellets were composed of (per $100 \mathrm{~g}$ ) $4 \mathrm{~g}$ of fat, $20 \mathrm{~g}$ of proteins, and $66 \mathrm{~g}$ of carbohydrates (UAR, Epinay sur Orge, France). This diet contained $3.90 \mathrm{kcal} / \mathrm{g}$, including $0.37 \mathrm{kcal} / \mathrm{g}$ from fat. All experiments were performed in accordance with the Principles of Laboratory Animal Care (National Institutes of Health publication 86-23, revised 1985) and the French national laws.

Behavioral phase shifts. To determine circadian rhythm of locomotor activity, wheel running was continuously recorded in 24 mice (experiment 1 ) and collected every 5 min as described previously (Challet et al., 2003). Mice were transferred to DD during 1 week to assess the endogenous period $(\tau)$ of each animal ( $\chi^{2}$ periodogram; ClockLab software; Actimetrics, Evanston, IL). Mice were then returned to LD with food available ad libitum for 2 weeks. Daily food intake was determined to be $5.4 \mathrm{~g}$ of chow. As in previous studies (Challet et al., 1998), mice were then randomly divided into three groups. The normocalorie-fed group received $100 \%$ of the mean daily food intake (i.e., $5.4 \mathrm{~g}$ ) $6 \mathrm{~h}$ after the onset of light (i.e., at ZT6). The hypocalorie-fed group was given 66\% (i.e., $3.6 \mathrm{~g}$ ) of the daily food intake at ZT6. A third group of mice fed ad libitum served as control, with a mean spontaneous food intake of $5.4 \mathrm{~g}$. The period of food restriction lasted 3 weeks. Thereafter, the mice were transferred to DD and fed ad libitum for 2 weeks. On the day of transfer to DD, lights were not switched on at pZT0, and all of the animals received food ad libitum from pZT6. Body mass was measured weekly.

For assessment of phase changes in the SCN-controlled nocturnal period of activity, the onsets of nocturnal locomotor activity were determined during the last $8 \mathrm{~d}$ of ad libitum baseline conditions under LD and from the second to ninth day of ad libitum (re)feeding in DD (ClockLab). To determine individual phase changes, a linear regression analysis of the onsets of activity (ClockLab) was performed by projecting the onset phase of the free run in DD back to the mean onset phase under LD baseline conditions.

For assessment of the daily pattern of wheel-running activity, the cumulative wheel revolutions performed during eight $3 \mathrm{~h}$ intervals (ClockLab) starting at ZT0 were determined during the last $8 \mathrm{~d}$ of ad libitum baseline conditions under LD and the last $8 \mathrm{~d}$ of food restriction under LD. Daily activity was defined as the total wheel revolutions per day averaged for each mouse over these two $8 \mathrm{~d}$ periods.

For assessment of the day-night pattern of food intake, food intake was measured by weighing remaining food pellets with a scale (Fisher Bioblock Scientific, Illkirch, France) at the nearest $0.1 \mathrm{~g}$ during $12 \mathrm{~h}$ daytime and $12 \mathrm{~h}$ nighttime over $24 \mathrm{~h}$ at the end of ad libitum baseline conditions under LD. During the third week of food restriction, food intake was measured over $24 \mathrm{~h}$ during five $3 \mathrm{~h}$ intervals from ZT0 to ZT15 and one $9 \mathrm{~h}$ interval from ZT15 to ZT24.

Circadian oscillations of clock genes. In experiment 2, mice were exposed to 3 weeks of food restriction under LD conditions as described for experiment 1 and were then transferred to DD (i.e., lights were not switched on at pZT0). On this day without light, mice were killed at $3 \mathrm{~h}$ intervals, starting at projected time of lights on (i.e., pZT0; $n=4$ per time point for a given feeding condition). Normocalorie-fed and hypocaloriefed animals (except those already killed at pZT0, pZT3, and pZT6) were provided with respective diets at pZT6, whereas control mice had food available ad libitum. After isofluorane anesthesia and decapitation, brains were removed and stored at $-80^{\circ} \mathrm{C}$. Trunk blood was sampled to determine blood glucose (Glucotrend premium kit; Roche Diagnostics, Meylan, France). Antisense and sense RNA probes were generated with an in vitro transcription kit (Maxiscript; Ambion, Austin, TX). Here we used riboprobes of $r$ Per $1, m P e r 2, m C r y 2$ (from plasmids kindly provided by Dr. H. Okamura, University of Kobe, Kobe, Japan), rVasopressin (AVP) (Dardente et al., 2002), and mBmal1b. mBmal1b cDNA fragments were PCR amplified using the following oligonucleotides: 5'-GCCCCACCGACCTACTCT- $3^{\prime}$ and 5'-CATCGTTACTGGGACTACTTGAT-3' (nucleotides 55-1807 of GenBank accession number AB012601). mBmal1b PCR product of the expected size was cloned into the PCRScript SK $(+)$ cloning vector (Stratagene, Amsterdam, The Netherlands). Identity and orientation of the cloned PCR fragment was confirmed by sequencing (AGOWA Sequencing Services, Berlin, Germany). Brain sections $(14 \mu \mathrm{m})$ were postfixed in $4 \%$ phosphate-buffered paraformaldehyde, rinsed with PBS and SSC, and then acetylated twice in $0.1 \mathrm{M}$ triethanol-amine, washed again with SSC and PBS, treated with $0.1 \mathrm{M}$ Tris containing glycine, rinsed with SSC and PBS, and dehydrated in a graded ethanol series. Sections were hybridized overnight with either denatured antisense or sense riboprobe in a humid chamber at $62^{\circ} \mathrm{C}$. Sections were then rinsed with SSC, treated with ribonuclease A (Sigma, St. Louis, $\mathrm{MO}$ ), rinsed with stringency washes of SSC, and dehydrated in a graded ethanol series. Slices and radioactive standards were exposed for 1 week to an autoradiographic film [Biomax MS-1 from Eastman Kodak (Rochester, NY); Sigma]. Standards were included in each cassette to verify that the measured values of optical densities were in the linear response range of the film. Densitometric analysis of hybridization signals was performed with a computerized analysis system (RAG200; Biocom, Les Ulis, France). The optical density of specific signal was calculated by subtracting the intensity of staining background area (defined as a circle of 100 $\mu \mathrm{m}$ diameter) measured in the anterior hypothalamic area above the SCN from that of a circle of $100 \mu \mathrm{m}$ diameter measured in the right and left SCN. Measures were made on three consecutive slices in the rostrocaudal middle of the SCN and averaged for a given brain. Data were expressed as relative optical density values.

Light-induced behavioral phase shifts. Experiment 3 was designed to test the ability of timed calorie restriction to modulate light-induced phase shifts of locomotor activity rhythm. Mice were exposed to 3 weeks of food restriction under LD conditions and then transferred to DD, as described for experiment 1 (hypocalorie fed vs ad libitum fed), except that, on the first day of DD, animals were placed for $30 \mathrm{~min}$ in a white chamber delivering a light pulse of 100 lux at $3 \mathrm{~h}$ intervals starting at projected ZT0 (five hypocalorie-fed mice and four ad libitum-fed mice per time point). Six hypocalorie-fed and six ad libitum-fed mice that were placed into a dark chamber (0 lux) for 30 min served as dark controls. Assessment of light-induced phase changes in the SCN-controlled nocturnal period of activity followed the method described above for determining possible behavioral phase changes in response to restricted feeding.

Responses to light of pineal melatonin and clock genes. In experiment 4, mice were exposed to 3 weeks of food restriction under LD conditions and then transferred to DD, as described for experiment 1 (hypocalorie fed vs ad libitum fed). On the first day of DD, food was provided at the usual time, and then subgroups of animals were exposed to a light pulse at projected ZT0, ZT3, ZT6, ZT9, ZT12, ZT15, ZT18, or ZT21. Lightexposed mice (four hypocalorie fed mice and four ad libitum-fed mice 
per time point) were killed in darkness $60 \mathrm{~min}$ after the beginning of $30 \mathrm{~min}$ light exposure, and dark controls (four hypocalorie-fed mice and three ad libitum-fed mice per time point) were killed at the same time in darkness. After isofluorane anesthesia and decapitation, brains and pineal glands were removed and stored at $-80^{\circ} \mathrm{C}$. Pineal melatonin content was determined by radioimmunoassay, as described previously (Vivien-Roels et al., 1999). In situ hybridization with riboprobes for Per1 and Per2 was performed as above on hypothalamic slices. Plasmids were kindly provided by Dr. $\mathrm{H}$. Okamura.

Statistical analysis. Data are presented as means \pm SEM. ANOVAs, with or without repeated measures, were followed by post hoc comparisons with the Student-Newman-Keuls test. For a given feeding condition, the daily patterns of gene expression and pineal melatonin were fitted by a nonlinear least-squares regression (SigmaPlot software; Jandel Scientific, Chicago, IL) to determine the basal level, the mean peak level above basal, and the phase and duration of the peak (i.e., the interval between half-maximal values on either side of the peak) using the following logistic equation: $y=y_{0}+$ $\left(y_{\max } /((1+\exp (\right.$ slope $1 \times(\phi-x))) \times$ $(1+\exp ($ slope $2 \times(x-\phi-d)))))$, where $y$ is the level of mRNA, $y_{0}$ is the basal level, $y_{\max }$ is the mean peak level above basal, slope 1 is the ascending slope, $\phi$ is the time of half increase, slope 2 is the descending slope, and $d$ is the duration of the peak (i.e., the delay between times of half-amplitude on increase and decrease). The slopes were determined from regression on a first trial run and then set at constant values throughout. The effects of the feeding conditions (ad libitum feeding vs normocaloric diet, ad libitum feeding vs hypocaloric diet, and normocaloric vs hypocaloric diet) were then assessed by a variance-covariance analysis in which the following parameters were introduced stepwise according to F-to-enter: $\Delta_{y 0}=$ difference in basal level, $\Delta_{y \max }=$ difference in amplitude, $\Delta_{\phi}=$ difference in phase (delay or advance of time of half-increase), and $\Delta_{d}=$ difference in peak duration. When all parameters had been added, the final equation was as follows: $y=\left(y_{0}+\Delta_{\mathrm{y} 0}\right)+\left(\left(y_{\max }+\Delta_{\text {max }}\right) /((1+\right.$ $\exp \left(\right.$ slope $\left.\left.1 \times\left(\phi+\Delta_{\phi}-x\right)\right)\right) \times\left(1+\exp \left(\right.\right.$ slope $2 \times\left(x-\phi-\Delta_{\phi}-d-\right.$ $\left.\left.\left.\left.\Delta_{d}\right)\right)\right)\right)$.

The analysis gave the calculated values of $\Delta_{y 0}, \Delta_{y \max }, \Delta_{\phi}$, and $\Delta_{d}$ and the associated probabilities. Any effect was considered statistically significant if $p \leq 0.05$.

\section{Results}

\section{Changes in daily pattern of wheel-running activity}

At a behavioral level, hypocalorie-fed mice showed changes in their daily pattern of locomotor activity, characterized by a robust bout of activity before the time of feeding and an alteration of the phase lock of the nocturnal activity to dark onset (Fig. 1). Normocalorie-fed mice expressed a small bout of activity before the time of feeding and no apparent change in the timing of nocturnal activity (Fig. 1). Phase changes of the SCN-controlled rhythm of locomotor activity were assessed during ad libitum refeeding in DD. Although both control mice fed ad libitum and mice fed with a normocaloric diet showed very small behavioral phase shifts $(0.1 \pm 0.1$ and $0.6 \pm 0.2 \mathrm{~h}$, respectively), the phase of the activity onset was advanced by $3.6 \pm 0.4 \mathrm{~h}$ in hypocalorie-fed mice $(p<0.001)$ (Fig. 1$)$.

The endogenous period in DD was not significantly modified by the treatment (baseline vs after food restriction, $\tau=23.8 \pm$ 0.04 vs $23.9 \pm 0.05 \mathrm{~h}$, respectively; $p>0.05$ ), nor by the feeding condition (hypocaloric feeding, normocaloric feeding, and ad libitum feeding, $\tau=23.8 \pm 0.05,23.8 \pm 0.04$, and $23.9 \pm 0.07 \mathrm{~h}$, respectively; $p>0.05$ ) (Fig. 1 ), and the treatment $\times$ feeding condition interaction was not significant $(p>0.05)$.

During baseline, all mice show a large increase of wheelrunning activity at night (Fig. $2 A$ ). The average total number of wheel revolutions during days of baseline was similar between the three nutritional groups $(28426 \pm 2103,30930 \pm 2643$, and $29377 \pm 2282$ wheel revolutions in hypocalorie-fed mice, normocalorie-fed mice, and control mice fed ad libitum, respectively; $p>0.1$ ). In response to food restriction, the average total number of wheel revolutions was not significantly modified by the nutritional status $(31449 \pm 2639,28648 \pm 2506$, and $25216 \pm$ 1552 wheel revolutions in hypocalorie-fed, normocalorie-fed, and control ad libitum-fed mice, respectively; $p>0.1$ ). However, both hypocalorie- and normocalorie-fed mice displayed changes in their day-night pattern of activity compared with their activity pattern during baseline and to the pattern of activity in control mice fed ad libitum. The changes of activity pattern were more marked in hypocalorie-fed than in normocalorie-fed mice (Figs. $1,2 \mathrm{~A})$. During the $6 \mathrm{~h}$ interval before the time of providing food to normocalorie- and hypocalorie-fed mice (i.e., from ZT0 to ZT6), the so-called food-anticipatory bout of activity was much larger in the latter group. During the two $3 \mathrm{~h}$ intervals after mealtime (i.e., from ZT6 to ZT12), both groups of mice displayed a higher level of locomotor activity than that performed at the same time by control mice. During the first $3 \mathrm{~h}$ nocturnal interval (ZT12-ZT15), locomotor activity was slightly decreased in normocalorie-fed and ad libitum-fed mice, although it was increased in hypocalorie-fed mice compared with respective baseline values. In the last $3 \mathrm{~h}$ nocturnal interval (ZT21-ZT24), the level of wheel-running activity was significantly reduced in normocalorie-fed mice and, to a larger extent, in hypocalorie-fed mice (Fig. 2A).

Changes in day-night pattern of food intake, body mass, and $24 \mathrm{~h}$ blood glucose

Daytime and nighttime food intake was modified significantly by time (baseline vs food restriction; $p<0.001$ ) and feeding condi- 

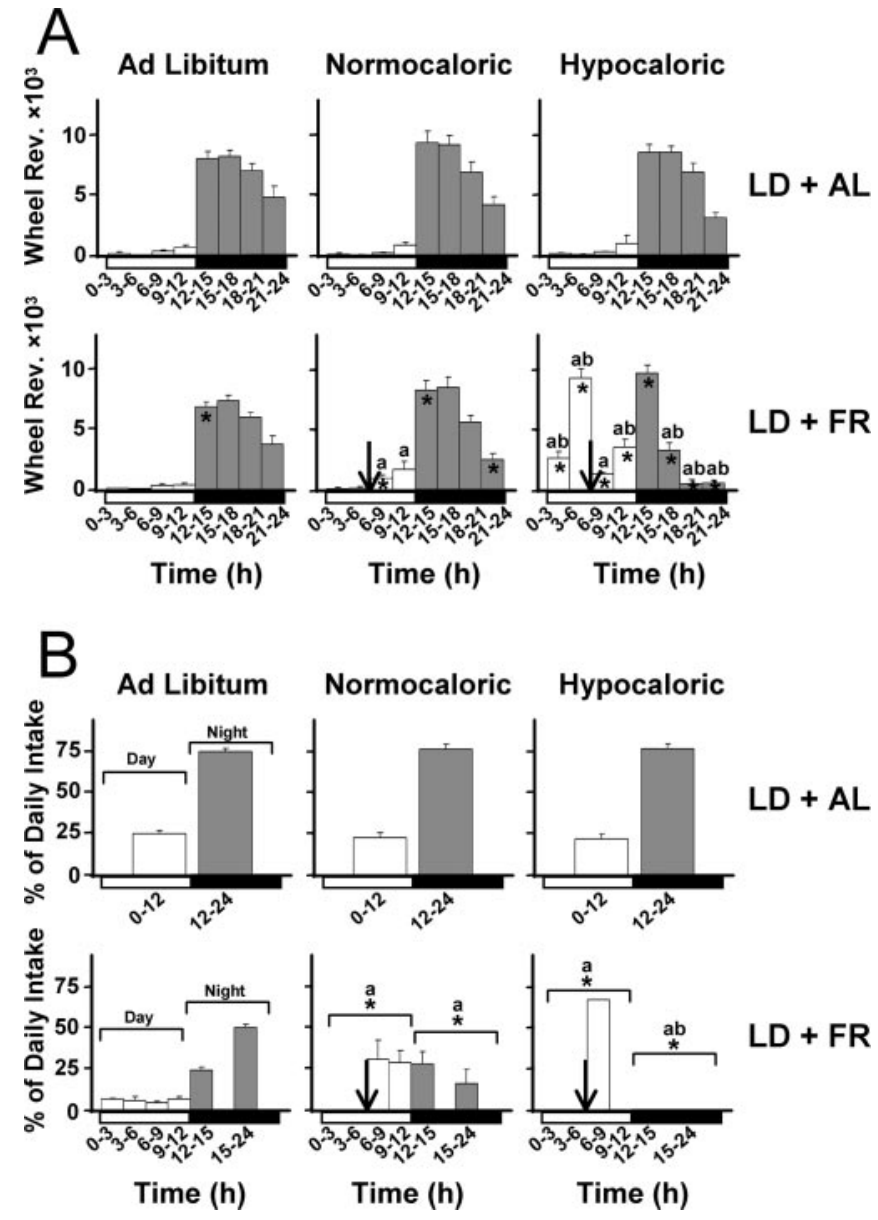

Figure 2. A, Daily pattern of wheel-running activity during the last $8 \mathrm{~d}$ in light/dark cycle and ad libitum feeding (LD $+\mathrm{AL}$; top row) and during the last $8 \mathrm{~d}$ in light/dark cycle and food restriction (LD + FR; bottom row). Wheel-running activity is presented as cumulated wheel revolutions (Rev.) during $3 \mathrm{~h}$ intervals. ${ }^{*} p<0.05$ in bouts of activity for a given nutritional status between $L D+A L$ and $L D+F R ;{ }^{a, b} p<0.05$ in activity bouts in hypocalorie-fed or normocalorie-fed mice compared with ad libitum-fed mice and in hypocalorie-fed versus normocalorie-fed mice, respectively. Nighttime is indicated by a black bar on abscissa. Time of hypocaloric and normocaloric feeding is indicated by a vertical arrow $6 \mathrm{~h}$ after lights on. Means \pm SEM ( $n=8$ per feeding condition). $B$, Daily pattern of food intake (expressed as percentage of daily intake during baseline) in light/dark cycle and ad libitum feeding ( $L D+A L$; top row) and during the third week of food restriction under a light/dark cycle (LD + FR; bottom row). Food intake during baseline was determined over $24 \mathrm{~h}$ at two $12 \mathrm{~h}$ intervals, daytime and nighttime. Food intake during food restriction was measured over $24 \mathrm{~h}$, every $3 \mathrm{~h} \mathrm{interval} \mathrm{from}$ ZT0 to ZT15, and during a $9 \mathrm{~h}$ interval from ZT15 to ZT24. During food restriction, the normocalorie-fed group received $100 \%$ of daily food intake (i.e., $5.4 \mathrm{~g}$ ) $6 \mathrm{~h}$ after the onset of light, and they ate 60 and $40 \%$ of this diet during the afternoon (i.e., from ZT6 to ZT12) and the night (i.e., from ZT12 to ZT24), respectively. The hypocalorie-fed group was given only $66 \%$ of baseline food intake (i.e., $3.6 \mathrm{~g}$ ) at ZT6. This hypocaloric diet was eaten between ZT6 and ZT9. Control mice had ad libitum access to food during the experiment. Statistical analysis of food intake was performed on the cumulated intake during daytime and nighttime. ${ }^{*} p<0.05$ in diurnal or nocturnal food intake for a given nutritional status between $L D+A L$ and $L D+F R$; ${ }^{a, b} p<0.05$ in diurnal or nocturnal food intake in hypocalorie-fed or normocalorie-fed mice compared with ad libitum-fed mice and in hypocalorie-fed versus normocalorie-fed mice, respectively. Nighttime is indicated by a black bar on abscissa. Time of hypocaloric and normocaloric feeding is indicated by a vertical arrow $6 \mathrm{~h}$ after lights on. Means \pm SEM ( $n=6$ per feeding condition).

tion (hypocaloric feeding, normocaloric feeding, and ad libitum feeding; $p<0.05$ ). Moreover, the time $\times$ feeding condition interaction was significant $(p<0.001)$. During baseline, all mice ate 25 and $75 \%$ of their spontaneous daily food intake during daytime and nighttime, respectively $(p>0.1)$ (Fig. $2 B$ ). In re-
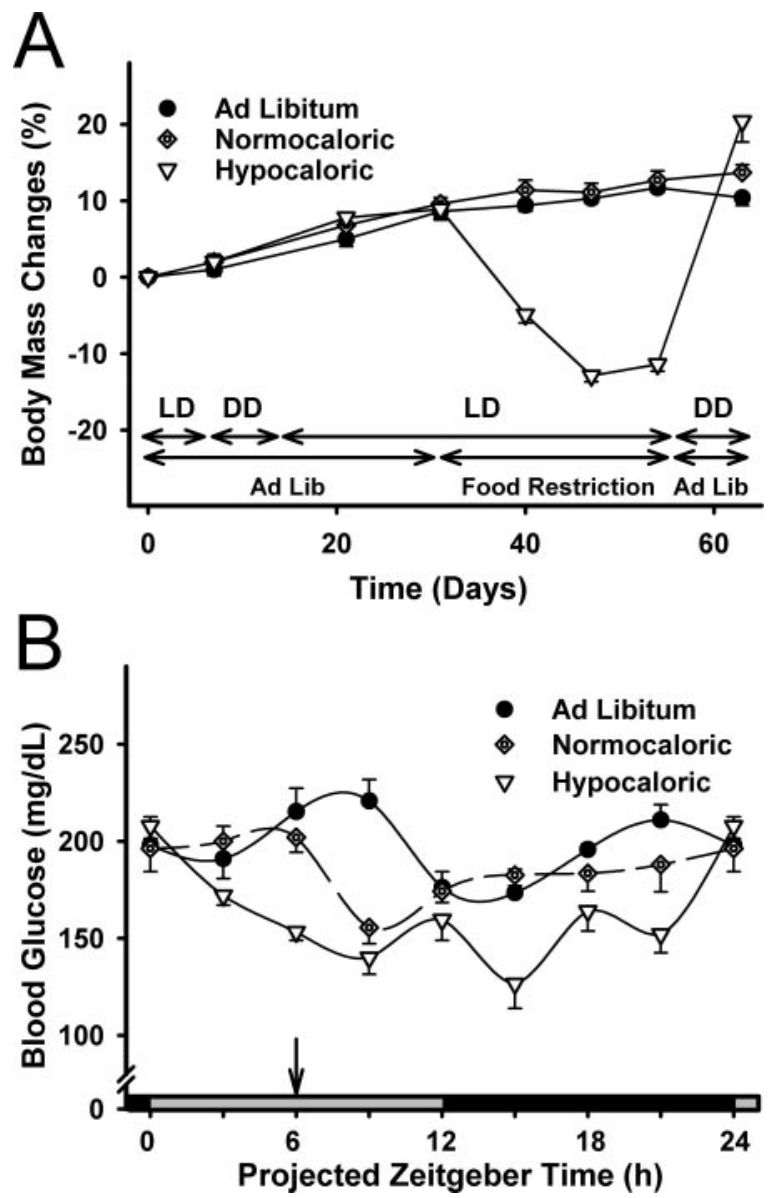

Figure 3. $A$, Changes in body mass (expressed as percentage of initial body mass at day 0 ) in hypocalorie-fed, normocalorie-fed, and ad libitum-fed mice. B, Daily changes of blood glucose in hypocalorie-fed, normocalorie-fed, and ad libitum-fed mice. pZT0 and pZT12 are defined, respectively, as the projected times of lights on and lights off in the previous lighting cycle. Data for pZT0 are double plotted at pZT24. Time of hypocaloric and normocaloric feeding $6 \mathrm{~h}$ after lights on is indicated by a vertical arrow.

sponse to food restriction, hypocalorie-fed mice ate their diet ( $66 \%$ of baseline intake) within the first $3 \mathrm{~h}$ interval after food was provided (i.e., between ZT6 and ZT9). Normocaloric feeding led to a progressive change in the day/night ratio of food intake. During the third week of food restriction, daytime food intake in normocalorie-fed mice ( $61 \pm 11 \%$ of daily intake) was larger than that of ad libitum-fed controls ( $23 \pm 2 \%$ of daily intake) and not different from that of hypocalorie-fed mice $(66 \pm 0 \%$ of baseline intake) (Fig. $2 \mathrm{~B}$ ). Nocturnal food intake was lower in normocalorie-fed mice ( $39 \pm 11 \%$ of daily intake) than in ad libitum-fed controls ( $77 \pm 2 \%$ of daily intake) (Fig. $2 B$ ).

Body mass was altered significantly by feeding condition (hypocaloric feeding, normocaloric feeding, and ad libitum feeding; $p<0.001)$ and the 3 weeks of food restriction $(p<0.001)$ (Fig. $3 A$ ). Body mass decreased only in mice fed with hypocaloric diet (after an initial loss during the first and second week, amounting to $-13 \pm 2$ and $-20 \pm 1 \%$ of body mass measured at the end of baseline period, respectively, it remained stable at $-19 \pm 1 \%$ during the third week of food restriction). During the same 3 week period, mice fed ad libitum or with a normocaloric diet slightly increased body mass $(3 \pm 1$ and $3 \pm 1 \%$ of body mass measured at the end of baseline period, respectively). The loss of body mass in hypocalorie-fed mice was fully recovered by 1 week of ad libitum refeeding (Fig. 3A). 
Blood glucose was modified significantly by feeding condition $(p<0.001)$ and time of the day $(p<0.001)$. The decrease of daily blood glucose was much more marked in hypocalorie-fed mice $(-19 \%)$ than in normocalorie-fed animals $(-6 \%)$ compared with control values $(159.4 \pm 4.9,185.3 \pm 4.3$, and $197.7 \pm 4.6$ $\mathrm{mg} / \mathrm{dl}$, respectively) (Fig. $3 B$ ).

\section{Changes in expression of clock and clock-controlled genes}

At a molecular level, the daily pattern of expression was assessed for four clock genes (i.e., Per1, Per2, Cry2, and Bmal1) and one clock-controlled gene (i.e., $A V P$ ) across feeding condition. The daily expression of the mRNA studied was very similar between normocalorie-fed mice and control animals fed ad libitum, except that the amplitude of Per1 mRNA was slightly but significantly higher in the SCN of normocalorie-fed mice $(p<$ 0.05) (Figs. 4, 5). In contrast, a number of differences in gene expression were detected in hypocalorie-fed mice compared with control ad libitum-fed or normocalorie-fed mice. With respect to expression of SCN clock genes, the daily oscillation of Per1 in hypocalorie-fed mice was damped compared with that in ad libitum-fed mice $(p<0.05)$ and normocalorie-fed mice $(p<$ $0.05)$. SCN Per1 oscillation in hypocalorie-fed mice was phase advanced by $1.4 \pm 0.4$ and $1.1 \pm 0.4 \mathrm{~h}$ compared with that in $\mathrm{ad}$ libitum-fed $(p=0.001)$ and normocalorie-fed $(p<0.01)$ mice, respectively. Daily oscillation of Per 2 in the SCN of hypocaloriefed mice was slightly, but not significantly, phase advanced compared with ad libitum-fed $(0.6 \pm 0.3 \mathrm{~h} ; p=0.07)$ and normocalorie-fed $(0.3 \pm 0.4 \mathrm{~h} ; p>0.1)$ mice. Moreover, in addition to a higher amplitude $(p=0.04)$ compared with ad libitum-fed but not with normocalorie-fed mice, the phase of Cry 2 mRNA was phase advanced by $3.3 \pm 0.8 \mathrm{~h}$ in hypocaloriefed versus ad libitum-fed mice $(p<0.001)$ and by $2.3 \pm 0.8 \mathrm{~h}$ in hypocalorie-fed versus normocalorie-fed mice $(p<0.01)$. In contrast, the daily profile of Bmal1 was similar regardless of feeding condition (Fig. 4). Finally, the phase of the daily expression of SCN AVP was phase advanced by $4.5 \pm 0.5 \mathrm{~h}$ in hypocalorie-fed versus ad libitum-fed mice $(p<0.001)$ and by $2.8 \pm 0.5 \mathrm{~h}$ in hypocalorie-fed versus normocalorie-fed mice $(p<0.001)$.

\section{Changes in circadian rhythm and light-induced suppression of pineal melatonin}

Logarithmic transformation of individual values of pineal melatonin was performed before comparisons to ensure homogeneity of residual variances. The daily rhythm of pineal melatonin synthesis was phase advanced in hypocalorie-fed compared with that of ad libitum-fed mice ( $p<0.001)$ (Fig. 6). The magnitude of the phase advance was larger for the onset (i.e., $3.2 \mathrm{~h}$ ) compared with the offset (i.e., 1.3 h) of melatonin synthesis. Light-induced suppression of pineal melatonin assessed over the circadian cycle was not significantly modified by the nutritional status (hypocaloric diet vs ad libitum food; $p>0.1$ ) (Fig. 6).

\section{Altered phase-shift responses of locomotor activity rhythm to light}

The amplitude and direction of light-induced phase shifts depended on the time of light exposure $(p<0.0001)$ (Figs. 7, 8). The phase-response curve to light in control $\mathrm{C} 3 \mathrm{H}$ mice fed ad libitum was characterized by phase-delaying and phaseadvancing regions in the early (pZT12-pZT15) and late (pZT18pZT24) subjective night, respectively. During most of the subjective day (pZT0-pZT6), light exposure led to negligible behavioral phase shifts in control mice. Unexpectedly, compared with other phase-response curves to light in mice (Schwartz and Zimmerman, 1990), phase advances were apparent at pZT9 in $\mathrm{C} 3 \mathrm{H}$ mice fed ad libitum. The shape of the phase-response curve to light was dramatically changed in hypocalorie-fed mice $(p<$ 0.0001) (Figs. 7, 8). Whereas light-induced phase delays were slightly reduced, phase advances in late night were more than twice in hypocalorie-fed mice compared with those in ad libitumfed animals. Moreover, large phase advances were still detectable in hypocalorie-fed mice exposed to a light pulse during the subjective day, a temporal window that corresponds to the "dead zone" of the phase-response curve to light in control animals fed ad libitum (Figs. 7, 8).

\section{Altered responses of clock gene expression to light}

In control animals fed ad libitum, light exposure during the subjective night in darkness led to a marked increase of Per1 mRNA levels in the SCN $(p<0.001)$ (Figs. 9, 10). Light induction of Per1 in the SCN of hypocalorie-fed mice was lower compared with respective levels in ad libitum fed mice $(p<0.001)$ (Figs. 9, $10)$. The reduction in light-exposed animals occurred not only during the night but also during the day (Fig. 10, top left), as confirmed with a nonsignificant feeding condition $\times$ time interaction $(p>0.5)$. When compared with respective dark controls, the reduction of light induction of Perl in hypocalorie-fed mice was detected during the night (Fig. 10, top right). Light induction of Per 2 was also altered in the SCN of hypocalorie-fed mice compared with that in control mice fed ad libitum (Figs. 9, 10). Contrary to Per1, there was an overall increased induction of Per2 by light exposure in hypocalorie-fed mice $(p<0.05)$ (Fig. 10, bottom left). The small increase of Per2 induction in the SCN of hypocalorie-fed mice was not time dependent compared with ad libitum-fed mice also exposed to light, given that feeding condition $\times$ time interaction was not significant $(p>0.5)$ (Fig. 10, bottom left). When compared with respective dark controls, a larger light induction of Per 2 was detected in the early night (Fig. 10, bottom right).

\section{Discussion}

To our knowledge, this is the first study in which a synchronizer is shown to compete with entrainment to $\mathrm{LD}$, both at the behav- 

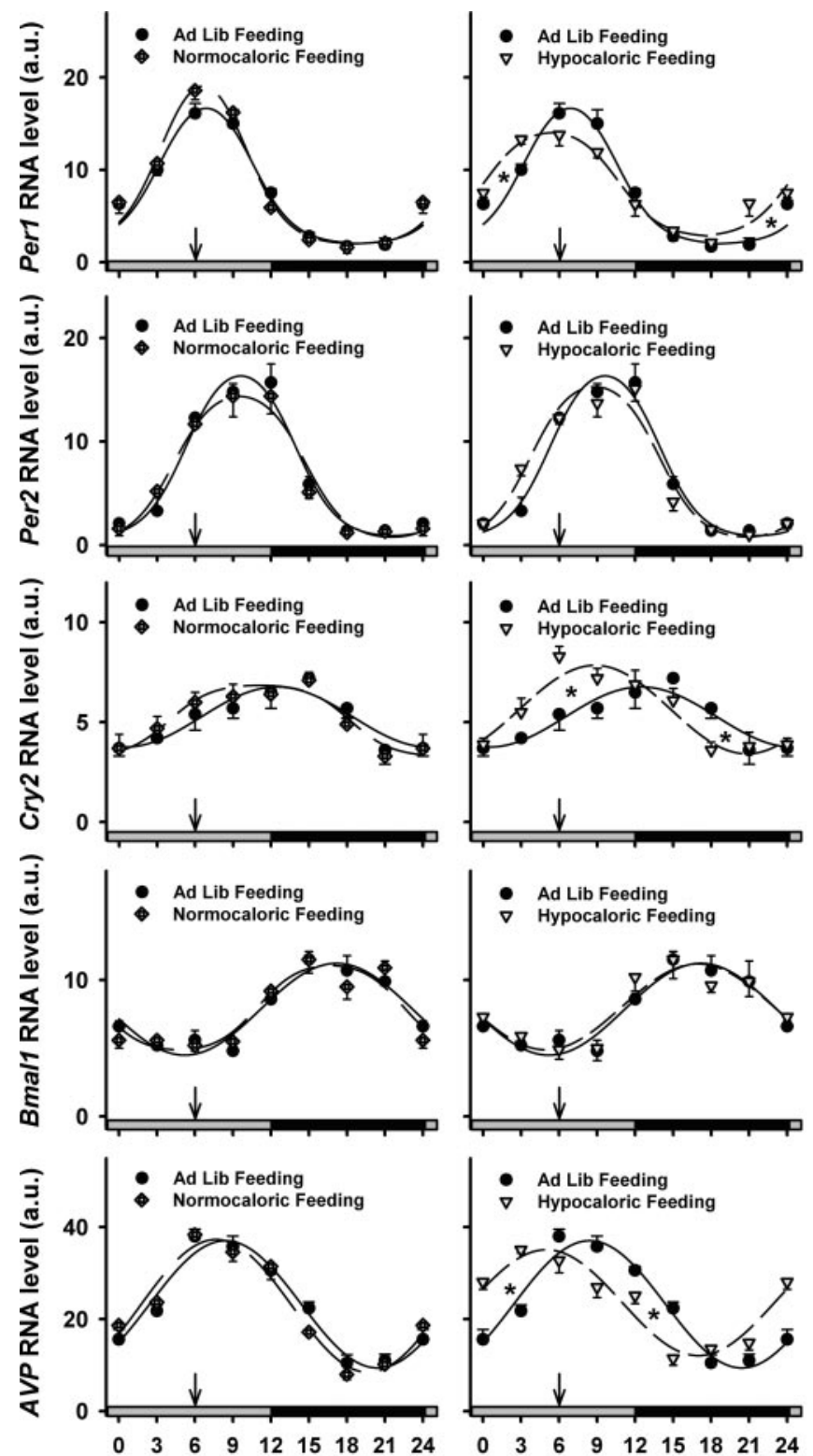

Projected Zeitgeber Time (h) Projected Zeitgeber Time (h)

Figure 5. Daily profiles of Per1, Per2, Cry2, Bmal1, and vasopressin (AVP) mRNA levels in the $\mathrm{SCN}$ of ad libitum-fed versus normocalorie-fed mice (left column) and of ad libitum-fed versus hypocalorie-fed mice (right column) and respective fitted curves. Means \pm SEM $(n=4$ per feeding condition at a given time point) and fitted curves. Asterisks indicate a significant phase shift between the two curves. Data for pZTO are double plotted at pZT24. Nighttime is indicated by a black bar on abscissa. Time of hypocaloric and normocaloric feeding is indicated by a vertical arrow $6 \mathrm{~h}$ after lights on. a.u., Arbitrary unit.

ioral and molecular levels. Contrary to daily normocaloric feeding, timed hypocaloric feeding modifies clock gene expression in the SCN and the phase of three outputs from the SCN (i.e., AVP mRNA oscillation, daily rhythms of locomotor activity, and pineal melatonin). Moreover, both temporal gating of lightinduced phase shifts and light induction of Perl are deeply modified with daily hypocaloric feeding. These results thus demonstrate that repetitive food-related/metabolic cues interact with entrainment of the SCN to light.

\section{No phase-shifting effect of normocaloric feeding}

Normocaloric feeding presented at midday did not modify the daily expression of clock genes in the SCN or the timing of two
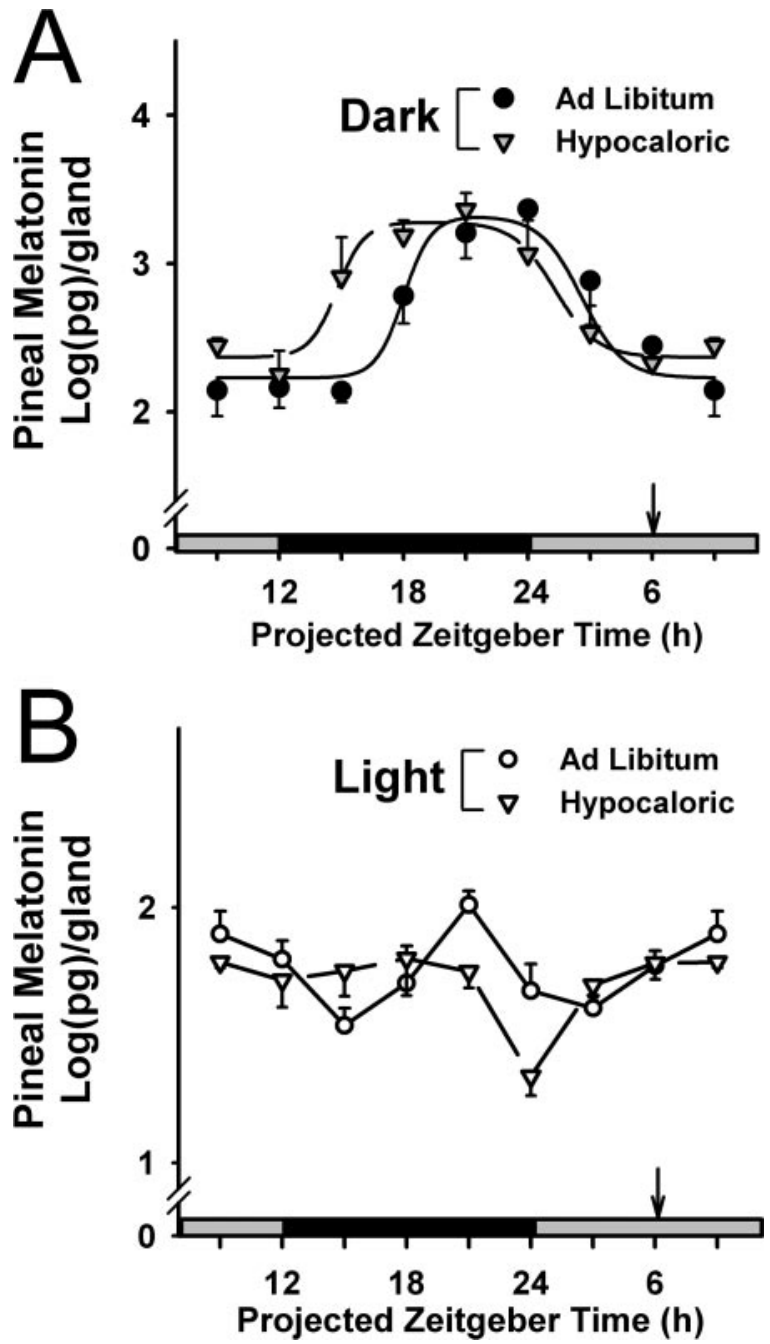

Figure 6. A, Daily rhythm of pineal melatonin in ad libitum-fed (filled circles; $n=3$ per time point) versus hypocalorie-fed (open triangles; $n=4$ ) mice and respective fitted curves. $B$, Light-induced suppression of plasma melatonin in ad libitum-fed ( $n=4$ per time point) versus hypocalorie-fed $(n=4)$ mice. Time of hypocaloric feeding is indicated by a vertical arrow $6 \mathrm{~h}$ after lights on. Data for pZT9 are double plotted.

circadian outputs, $A V P$ mRNA oscillation and locomotor activity rhythm. These variables were assessed in DD to prevent masking effects of light on clock-controlled parameters. Some changes, however, were detected on the daily pattern of wheel-running activity measured during normocalorie feeding under LD. In keeping with the increased daytime food intake and its nocturnal decrease, locomotor activity was increased in early afternoon (i.e., after mealtime) and reduced in late night. The lack of significant shift of activity rhythm in DD suggests that the changes in activity distribution under LD are not directly controlled by the SCN. Normocalorie feeding schedule also led to a post-prandial decrease of plasma glucose in late afternoon (Fig. 3). Although the ratio of daytime/nighttime intake is then less drastic than that in daytime restricted feeding (that is, a food access strictly restricted to daytime) as used by others (Damiola et al., 2000; Hara et al., 2001; Stokkan et al., 2001; Wakamatsu et al., 2001), neither normocaloric feeding nor daytime restricted feeding under LD affect clock gene expression within the SCN.

Phase-shifting effects of hypocaloric feeding

In contrast, timed hypocaloric feeding led to alterations in timing of the SCN clock: Per1 and Cry2 mRNA oscillations were phase 
advanced by 1.4 and $3.3 \mathrm{~h}$, respectively, whereas Per2 and Bmal1 oscillations remained essentially unchanged. Moreover, oscillation of AVP mRNA in the SCN, considered as a circadian output controlled by CLOCK/BMAL1 (Jin et al., 1999), was phase advanced by 4.5 h. Finally, when animals were transferred to DD, the onset of the nocturnal locomotor activity was phase advanced by $3.6 \mathrm{~h}$ in hypocaloriefed mice. The earlier nocturnal activity onset in calorie-restricted mice confirms previous findings (Challet et al., 1998; Holmes and Mistlberger, 2000; Sharma et al., 2000).

The behavioral phase advance in calorie-restricted mice cannot be ascribed to a shortening of $\tau$ because no changes were detected before versus after food restriction, nor can it be explained by transient hyperactivity it produces, although such bouts of activity during the subjective day can induce phase shifts of the SCN clock in nocturnal rodents (Mrosovsky et al., 1989; Van Reeth and Turek, 1989; Marchant et al., 1997). The food-anticipatory bout of activity that hypocalorie-fed rodents expressed before mealtime was initially considered to play a role in the behavioral phase advance. However, when food-anticipatory activity was prevented by daily immobilization, hypocaloric feeding was still capable of inducing a behavioral phase advance, indicating that foodanticipatory activity is not critical in mediating the phase-shifting effects of a hypocaloric diet (Challet et al., 1998). Furthermore, because mice fed ad libitum and stressed on a daily basis showed no behavioral phase advance, a daily stress cannot account for the shifting effects of hypocaloric feeding (Challet et al., 1998).

The distribution of wheel running during the active period affects the phase angle of photic synchronization (Mistlberger and Holmes, 2000). A higher amount of activity in early nocturnal period was associated with a 30 min advance in the phase angle of photic synchronization and a shortening of $\tau$. Therefore, the slight increased running activity detected in hypocalorie-fed mice in the first $3 \mathrm{~h}$ nocturnal period (and/or the activity decrease in the late night) may participate in the phase-advancing properties of timed hypocaloric feeding. However, hypocaloric feeding did not change $\tau$. Moreover, an activity decrease in late night, although to a lesser extent, was also observed in normocalorie-fed mice that did not show significant behavioral phase advances. Together, these data indicate that, if behavioral changes play a role in the phase-advancing effects of hypocaloric feeding, they cannot be the only factors involved, suggesting that metabolic factors are in that case predominant. Another finding supporting this metabolic hypothesis is that phase advances are induced whatever the time of the day (day or night) at which hypocaloric diet is given (Challet et al., 1998).

Serotonergic inputs from raphe nuclei and NPYergic projections from intergeniculate leaflets are neuronal pathways that have been shown to convey nonphotic signals to the SCN (Marchant et al., 1997). These pathways and projections from the ventromedial hypothalamic nuclei may also participate in transmitting nonphotic cues associated with hypocaloric feeding signals to the SCN (Challet and Pevet, 2003).

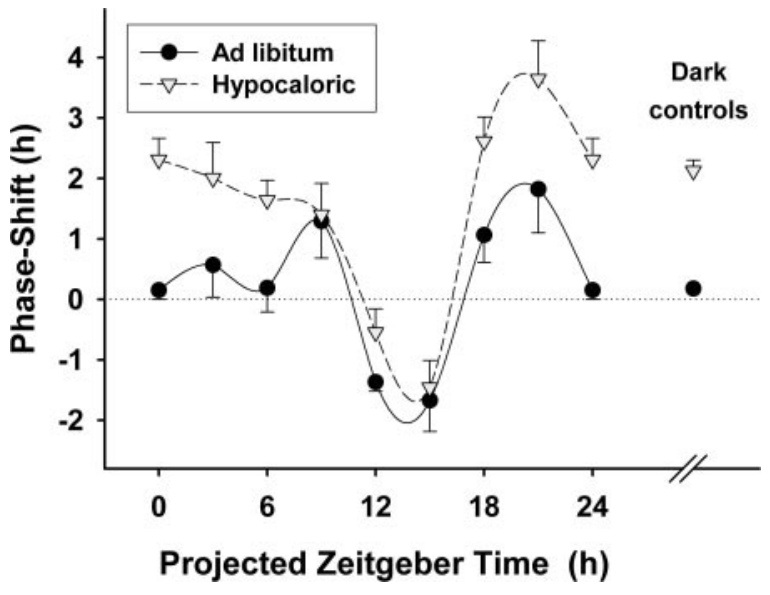

Figure 8. Phase-response curve of locomotor activity rhythm to light exposure in ad libitum-fed mice (filled circles; $n=4$ per time point) and hypocalorie-fed mice (open triangles; $n=5$ per time point). Six ad libitum-fed and six hypocalorie-fed mice not exposed to light served as "dark controls." Data for pZT0 are double plotted at pZT24.

\section{Effects of hypocaloric feeding on SCN gene expression}

Although three outputs studied (i.e., AVP expression, rhythms of locomotor activity, and pineal melatonin) displayed significant phase advances, there were discrepancies between the phase relationships of the clock genes studied in the SCN of hypocalorie-fed mice. Direct effects of food intake (i.e., masking effects) are unlikely to explain these various phase relationships in the SCN of animals that were still fed on the day they were killed because food intake per se does not affect clock gene expression in the SCN (Damiola et al., 2000; Stokkan et al., 2001). Transient alterations between oscillations of different clock genes (Reddy et al., 2002; Nagano et al., 2003) and between clock gene oscillation and behavioral outputs have been already reported after rapid changes in LD (Nagano et al., 2003; Vansteensel et al., 2003). Direct and conclusive implication of one or another clock gene in the control of behavioral outputs and the precise mechanisms leading to a 
Dark AL
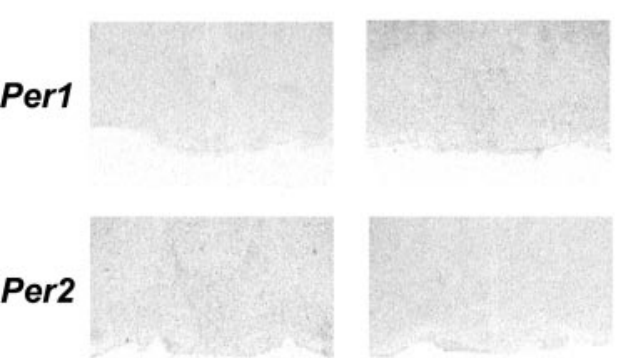

Light AL
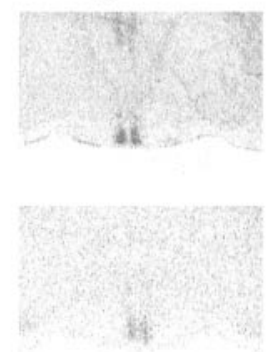

Figure 9. Expression of Per1 and Per2 in the suprachiasmatic nuclei of ad libitum-fed (AL) and hypocalorie-fed (HF) mice exposed to a $1 \mathrm{~h}$ light pulse (right rows) at pZT18 (Per1) and at pZT15 (Per2) or kept in dark at the same time points (left rows). Scale bar, $1 \mathrm{~mm}$.
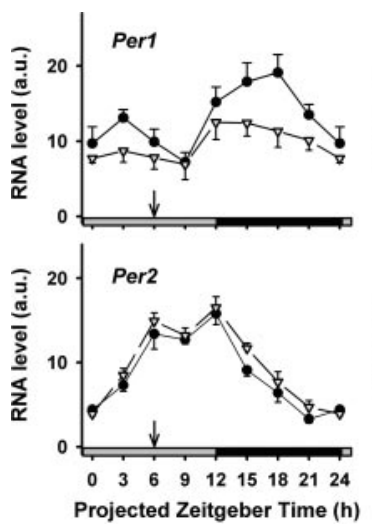
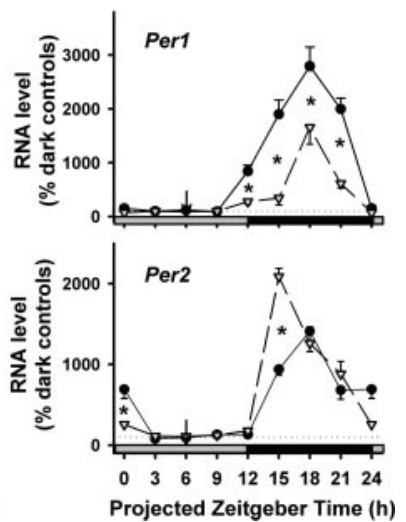

Figure 10. Altered light-induced expression of Per1 and Per2 in hypocalorie-fed mice (HF; open triangles) compared with control mice fed ad libitum (AL; filled circles). Left column, Data are presented as absolute values in light-exposed animals ( $n=4$ per time point per feeding condition). Right column, Data of light-exposed animals are presented as percentage of mRNA levels of mice not exposed to light (for each time point, 3 dark controls fed ad libitum and 4 dark controls previously fed with hypocaloric diet). Time of feeding during food restriction is indicated by a vertical arrow $6 \mathrm{~h}$ after lights on. a.u., Arbitrary unit. Data for pZT0 are double plotted at pZT24. The effect of feeding condition (AL vs HF) for Per1 and Per2 in light-exposed animals was significant at $p<0.001$ and $p<0.05$, respectively, whereas the interaction between feeding condition and time was not significant in both cases. ${ }^{*} p<0.05$ according to feeding conditions for a given time point.

complete and stable rephasing of clock genes still await clarification.

Whereas Per1 and Cry2 mRNA levels were phase advanced, the daily oscillation of Per 2 and Bmall in calorie-restricted mice matched closely that of control fed animals. When the photic Zeitgeber is changed by lengthening or shortening the photoperiod, the phase and duration of Per1 expression in the SCN are markedly modified accordingly, whereas Bmall expression remains centered to the dark period with no change in duration. This difference observed in both hamsters (Tournier et al., 2003) and sheep (Lincoln et al., 2002) indicates that the duration of Bmall oscillation is kept constant and its timing is phase locked to the middle of the nighttime when parameters of the photic synchronizer (photoperiod) are modified. A differential effect between phasing of Per1 and Bmal1 is also observed here in the case of a competition between light and feeding/hypocaloric cues.

Because AVP transcription is driven by CLOCK/BMAL1 heterodimers (Jin et al., 1999), the lack of phase shift in Bmal1 transcription concomitant with a $4 \mathrm{~h}$ phase advance in AVP oscillation is puzzling. Calorie restriction may modify the timing of protein synthesis and/or phosphorylation, so that initiation of
Light HF
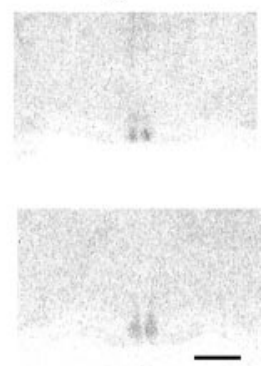

AVP transcription occurs earlier than normally imposed by photic synchronization. Alternatively, if Bmall oscillation is a phase marker of the SCN clock, the clock core may be considered in-phase in both calorie-restricted and ad libitum-fed mice, implying that the lag within the SCN concerns more specifically the temporal coupling of the clock core to the outputs.

Behavioral circadian responses to light Light exposure in the early and late subjective night induces subsequent phase delays and advances, respectively (Daan and Pittendrigh, 1976). Our aim was to understand the behavioral phase advance expressed in rodents fed with a hypocaloric diet and exposed to LD (Challet et al., 1997, 1998). The shift may result from competing food and light synchronizers on the circadian molecular loops. Alternatively, considering that a restricted feeding does not provide temporal cues to the SCN, the hypometabolic state in response to chronic calorie restriction may lead to a decreased suprachiasmatic sensitivity to light resetting. Indeed, low glucose availability can reduce circadian phase-shift responses to light (Challet et al., 1999). Because plasma glucose is decreased in hypocalorie-fed mice (Fig. 3B), we expected reduced lightinduced phase shifts with a hypocaloric feeding. Actually, lightresetting properties after timed calorie restriction are not decreased but are increased over the daily cycle. This finding thus suggests that decreased glucose availability cannot fully explain the data because, although light-induced phase delays were more or less reduced, light-induced phase advances were clearly increased. Moreover, there was no advance of the whole phaseresponse curve to light.

Stress does not affect light resetting (Meerlo et al., 1997; Challet et al., 2001) and thus cannot account for the altered responses to light attributable to hypocaloric feeding. Moreover, hypocaloric feeding led to changes in daily wheel-running activity. Spontaneous or triggered wheel-running activity generally reduces (Ralph and Mrosovsky, 1992; Mistlberger and Antle, 1998) or leaves unchanged light-induced phase shifts (Mistlberger and Holmes, 2000). Thus, a behavioral modulation does not appear to readily explain the increased photic resetting in hypocaloriefed mice. Nevertheless, the present data clearly indicate that the phase advances induced by hypocaloric restricted feeding may in part be explained by altered photic gating of the clock

\section{Molecular circadian responses to light}

Synchronization of the SCN to light is coupled with transcriptional mechanisms involving upregulation of Per mRNA levels. Light exposure activates expression of Per1 and Per2 in the SCN during the night, when light also induces behavioral phase shifts (Albrecht et al., 1997; Shigeyoshi et al., 1997).

In hypocalorie-fed mice, there was a clear reduction in the induction of Per 1 transcription by light exposure, whereas induction of Per2 was slightly increased. This differential effect supports the hypothesis that both genes play different functions in the synchronization of the SCN clock (Albrecht et al., 2001). For both genes, the effect in absolute values was not phase dependent but global over the daily cycle. As for the behavioral approach, these results suggest that timed calorie restriction leads to chronic (i.e., tonic) effects on the SCN clock.

Contrary to temporal restricted feeding, timed calorie restric- 
tion competes with entrainment to LD, as evidenced by altered circadian oscillations of clock and clock-controlled genes in the SCN and circadian responses to light. A better understanding of how calorie restriction impacts on the temporal patterns of transcriptional activity in the SCN cells will be useful to characterize the control of energy metabolism on circadian rhythmicity and its entrainment to light.

\section{References}

Abe H, Honma S, Namihira M, Tanahashi Y, Ikeda M, Honma KI (1998) Circadian rhythm and light responsiveness of BMAL1 expression, a partner of mammalian clock gene Clock, in the suprachiasmatic nucleus of rats. Neurosci Lett 258:93-96.

Albrecht U, Sun ZS, Eichele G, Lee CC (1997) A differential response of two putative mammalian circadian regulators, mPer1 and mPer2, to light. Cell 91:1055-1064.

Albrecht U, Zheng B, Larkin D, Sun ZS, Lee CC (2001) mPer1 and mPer2 are essential for normal resetting of the circadian clock. J Biol Rhythms 16:100-104.

Challet E, Pevet P (2003) Interactions between photic and nonphotic stimuli to synchronize the master circadian clock in mammals. Front Biosci $8: s 246-s 257$.

Challet E, Pevet P, Vivien-Roels B, Malan A (1997) Phase-advanced daily rhythms of melatonin, body temperature, and locomotor activity in foodrestricted rats fed during daytime. J Biol Rhythms 12:65-79.

Challet E, Solberg LC, Turek FW (1998) Entrainment in calorie-restricted mice: conflicting zeitgebers and free- running conditions. Am J Physiol 274:R1751-R1761.

Challet E, Losee-Olson S, Turek FW (1999) Reduced glucose availability attenuates circadian responses to light in mice. Am J Physiol 276:R1063-R1070.

Challet E, Turek FW, Laute MA, Van Reeth O (2001) Sleep deprivation decreases phase-shift responses of circadian rhythms to light in the mouse: role of serotonergic and metabolic signals. Brain Res 909:81-91.

Daan S, Pittendrigh CS (1976) A functional analysis of circadian pacemakers in nocturnal rodents. II. The variability of phase response curves. J Comp Physiol 106:253-266.

Damiola F, Le Minh N, Preitner N, Kornmann B, Fleury-Olela F, Schibler U (2000) Restricted feeding uncouples circadian oscillators in peripheral tissues from the central pacemaker in the suprachiasmatic nucleus. Genes Dev 14:2950-2961.

Dardente H, Poirel VJ, Klosen P, Pevet P, Masson-Pevet M (2002) Per and neuropeptide expression in the rat suprachiasmatic nuclei: compartmentalization and differential cellular induction by light. Brain Res 958:261-271.

Hara R, Wan K, Wakamatsu H, Aida R, Moriya T, Akiyama M, Shibata S (2001) Restricted feeding entrains liver clock without participation of the suprachiasmatic nucleus. Genes Cells 6:269-278.

Holmes MM, Mistlberger RE (2000) Food anticipatory activity and photic entrainment in food-restricted BALB/c mice. Physiol Behav 68:655-666.

Honma S, Kawamoto T, Takagi Y, Fujimoto K, Sato F, Noshiro M, Kato Y, Honma K (2002) Dec1 and Dec2 are regulators of the mammalian molecular clock. Nature 419:841-844.

Jin X, Shearman LP, Weaver DR, Zylka MJ, de Vries GJ, Reppert SM (1999) A molecular mechanism regulating rhythmic output from the suprachiasmatic circadian clock. Cell 96:57-68.

Kume K, Zylka MJ, Sriram S, Shearman LP, Weaver DR, Jin X, Maywood ES, Hastings MH, Reppert SM (1999) mCRY1 and mCRY2 are essential components of the negative limb of the circadian clock feedback loop. Cell 98:193-205.

Lincoln GA, Messager S, Andersson A, Hazlerigg D (2002) Temporal expression of seven clock genes in the suprachiasmatic nucleus and the pars tuberalis of the sheep: evidence for an internal coincidence timer. Proc Natl Acad Sci USA 99:13890-13895.

Marchant EG, Watson NV, Mistlberger RE (1997) Both neuropeptide Y and serotonin are necessary for entrainment of circadian rhythms in mice by daily treadmill running schedules. J Neurosci 17:7974-7987.

Meerlo P, van den Hoofdakker RH, Koolhaas JM, Daan S (1997) Stressinduced changes in circadian rhythms of body temperature and activity in rats are not caused by pacemaker changes. J Biol Rhythms 12:80-92.
Mistlberger RE (1994) Circadian food-anticipatory activity: formal models and physiological mechanisms. Neurosci Biobehav Rev 18:171-195.

Mistlberger RE, Antle MC (1998) Behavioral inhibition of light-induced circadian phase resetting is phase and serotonin dependent. Brain Res 786:31-38.

Mistlberger RE, Holmes MM (2000) Behavioral feedback regulation of circadian rhythm phase angle in light-dark entrained mice. Am J Physiol 279:R813-R821.

Mrosovsky N, Reebs SG, Honrado GI, Salmon PA (1989) Behavioural entrainment of circadian rhythms. Experientia 45:696-702.

Nagano M, Adachi A, Nakahama K, Nakamura T, Tamada M, MeyerBernstein E, Sehgal A, Shigeyoshi Y (2003) An abrupt shift in the day/ night cycle causes desynchrony in the mammalian circadian center. J Neurosci 23:6141-6151.

Preitner N, Damiola F, Lopez-Molina L, Zakany J, Duboule D, Albrecht U, Schibler U (2002) The orphan nuclear receptor REV-ERBalpha controls circadian transcription within the positive limb of the mammalian circadian oscillator. Cell 110:251-260.

Ralph MR, Mrosovsky N (1992) Behavioral inhibition of circadian responses to light. J Biol Rhythms 7:353-359.

Reddy AB, Field MD, Maywood ES, Hastings MH (2002) Differential resynchronization of circadian clock gene expression within the suprachiasmatic nuclei of mice subjected to experimental jet lag. J Neurosci 22:7326-7330.

Sato TK, Panda S, Miraglia LJ, Reyes TM, Rudic RD, McNamara P, Naik KA, FitzGerald GA, Kay SA, Hogenesch JB (2004) A functional genomics strategy reveals Rora as a component of the mammalian circadian clock. Neuron 43:527-537.

Schwartz WJ, Zimmerman P (1990) Circadian timekeeping in BALB/c and C57BL/6 inbred mouse strains. J Neurosci 10:3685-3694.

Sharma VK, Chidambaram R, Subbaraj R, Chandrashekaran MK (2000) Effects of restricted feeding cycle on the locomotor activity rhythm in the mouse Mus booduga. Physiol Behav 70:81-87.

Shearman LP, Sriram S, Weaver DR, Maywood ES, Chaves I, Zheng B, Kume K, Lee CC, van der Horst GT, Hastings MH, Reppert SM (2000) Interacting molecular loops in the mammalian circadian clock. Science 288:1013-1019.

Shigeyoshi Y, Taguchi K, Yamamoto S, Takekida S, Yan L, Tei H, Moriya T, Shibata S, Loros JJ, Dunlap JC, Okamura H (1997) Light-induced resetting of a mammalian circadian clock is associated with rapid induction of the mPer1 transcript. Cell 91:1043-1053.

Stephan FK (2002) The "other" circadian system: food as a Zeitgeber. J Biol Rhythms 17:284-292.

Stokkan KA, Yamazaki S, Tei H, Sakaki Y, Menaker M (2001) Entrainment of the circadian clock in the liver by feeding. Science 291:490-493.

Takahashi JS, Turek FW, Moore RY (2001) Circadian clocks, Vol 12, Handbook of behavioral neurobiology. New York: Kluwer Academic/Plenum.

Tournier BB, Menet JS, Dardente H, Poirel VJ, Malan A, Masson-Pévet M, Pévet P, Vuillez P (2003) Photoperiod differentially regulates clock genes' expression in the suprachiasmatic nucleus of Syrian hamster. Neuroscience 118:317-322.

Van Reeth O, Turek FW (1989) Stimulated activity mediates phase shifts in the hamster circadian clock induced by dark pulses or benzodiazepines. Nature 339:49-51.

Vansteensel MJ, Yamazaki S, Albus H, Deboer T, Block GD, Meijer JH (2003) Dissociation between circadian Perl and neuronal and behavioral rhythms following a shifted environmental cycle. Curr Biol 13:1538-1542.

Vivien-Roels B, Malan A, Rettori MC, Delagrange P, Jeanniot JP, Pevet P (1999) Daily variations in pineal melatonin concentrations in inbred and outbred mice. J Biol Rhythms 13:403-409.

Wakamatsu H, Yoshinobu Y, Aida R, Moriya T, Akiyama M, Shibata S (2001) Restricted-feeding-induced anticipatory activity rhythm is associated with a phase-shift of the expression of mPerl and mPer2 mRNA in the cerebral cortex and hippocampus but not in the suprachiasmatic nucleus of mice. Eur J Neurosci 13:1190-1196.

Zylka MJ, Shearman LP, Weaver DR, Reppert SM (1998) Three period homologs in mammals: differential light responses in the suprachiasmatic circadian clock and oscillating transcripts outside of brain. Neuron 20: $1103-1110$ 